\title{
Brief Study of Performance of Routing Protocols for Mobile Ad Hoc Networking in Ns-2
}

\author{
Gyanappa.A Walikar ${ }^{1}$, \\ ${ }^{1}$ Department of Comp. Sc. and Engg, Shivaji University, LNBCIET Raigoan (Satara), \\ Maharashtr, India
}

\author{
Dr. Sangappa Ramachandra Biradar ${ }^{2}$ \\ ${ }^{2}$ Department of Computer Science \& Engineering, Mody Institute of Technology and Science, \\ Lakshmangarh-332311, District: Sikar, \\ Rajasthan, INDIA
}

\begin{abstract}
Ad hoc networking allows portable devices to establish communication independent of a centralinfrastructure. However, the fact that there is no central Infrastructure and that the devices can move randomly gives rise to various kind of problems, such as routing and security. In this thesis the problem of routing is considered. This paper addresses issues pertaining to two different routing protocols Destination Sequenced Distance vector (DSDV) and Dynamic Source Routing (DSR) protocols, which are used for efficient routing under different scenarios in Mobile Ad-hoc Network (MANET), which plays a critical role in places where wired network are neither available nor economical to deploy. Objective of this paper to show the implementation of two routing protocols using Network Simulators and run it for different number of nodes. Then compared the two routing protocols for different network parameters and studied the efficient protocol under a particular scenario on the basis of two metrics. (1) Packet delivery ratio (2) Routing load DSDV is a Proactive gateway discovery algorithm where the gateway periodically broadcasts a gateway advertisement message which is transmitted after expiration of the gateways timer. DSR is a Reactive gateway discovery algorithm where a mobile device of MANET connects by gateway only when it is needed.
\end{abstract}

Keywords-DSDV, DSR, MANET, TMS, TTP.

\section{INTRODUCTION}

Wireless cellular systems have been in use since 1980s. We have seen their evolutions to first, second and third generation's wireless systems. Wireless systems operate with the aid of a centralized supporting structure such as an access point. These access points assist the wireless users to keep connected with the wireless system, when they roam from one place to the other. The presence of a fixed supporting structure limits the adaptability of wireless systems. In other words, the technology cannot work effectively in places where there is no fixed infrastructure. Future generation wireless systems will require easy and quick deployment of wireless networks. This quick network deployment is not possible with the existing structure of current wireless

Recent advancements such as Bluetooth introduced a new type of wireless systems known as mobile ad-hoc networks. Mobile ad-hoc networks or "short live" networks operate in the absence of fixed infrastructure. They offer quick and easy network deployment in situations where it is not possible otherwise. Ad-hoc is a Latin word, which means "for this or for this only." Mobile ad-hoc network is an autonomous system of mobile nodes connected by wireless links; each node operates as an end system and a router for all other nodes in the network. Nodes in mobile ad-hoc network are free to move and organize themselves in an arbitrary fashion. Each user is free to roam about while communication with others. The path between each pair of the users may have multiple links and the radio between them can be heterogeneous. This allows an association of various links to be a part of the same network. [1]

A mobile ad-hoc network is a collection of mobile nodes forming an ad-hoc network without the assistance of any centralized structures. These networks introduced a new art of network establishment and can be well suited for an environment where either the infrastructure is lost or where deploy an infrastructure is not very cost effective. The popular IEEE 802.11 "WI-FI" protocol is capable of providing ad-hoc network facilities at low level, when no access point is available. However in this case, the nodes are limited to send and receive information but do not route anything across the network. Mobile ad-hoc networks can operate in a standalone fashion or could possibly be connected to a larger network such as the Internet [1].

Mobile ad-hoc networks can turn the dream of getting connected "anywhere and at any time" in to reality. Typical application examples include a disaster recovery or a military operation. Not bound to specific situations, these networks may equally show better performance in other places. As an 


\section{Reactive Gateway Discovery}

Reactive routing technique is also known as on-demand routing. It takes a different approach of routing which overcomes the disadvantages of proactive routing. In reactive approaches those nodes which require connectivity to the Internet reactively find Internet gateways by means of broadcasting some kind of solicitation within the entire ad hoc network. This approach reduces the overhead of maintaining the route table as that of proactive. The node dynamically checks the route table, and if it does not find an entry for its destination or it finds an outdated entry it performs route discovery to find the path to its destination. [3] The signaling overhead is reduced in this method, particularly in networks with low to moderate traffic loads. However it has a drawback of route acquisition latency. That is when corresponding entry is not found the route discovery mechanism occurs which takes a very large amount of time, and for that time the packet waits for updation of the table.

\section{DSDV}

This protocol is based on classical Bellman-Ford routing algorithm designed for MANETS. Each node maintains a list of all destinations and number of hops to each destination. Each entry is marked with a sequence number. It uses full dump or incremental update to reduce network traffic generated by rout updates. The broadcast of route updates is delayed by settling time. The only improvement made here is avoidance of routing loops in a mobile network of routers. With this improvement, routing information can always be readily available, regardless of whether the source node requires the information or not. DSDV solve the problem of routing loops and count to infinity by associating each route entry with a sequence number indicating its freshness.

In DSDV, a sequence number is linked to a destination node, and usually is originated by that node (the owner). The only case that a non-owner node updates a sequence number of a route is when it detects a link break on that route. An owner node always uses even-numbers as sequence numbers, and a non-owner node always uses odd-numbers. With the addition of sequence numbers, routes for the same destination are selected based on the following rules: 1) a route with a newer sequence number is preferred; 2) in the case that two routes have a same sequence number, the one with a better cost metric is preferred [2]. The list which is maintained is called routing table. The routing table contains the All available destinations' IP address, Next hop IP address, Number of hops to reach the destination, Sequence number assigned by the destination node, Install time.

The sequence number is used to distinguish stale routes from new ones and thus avoid the formation of loops. The stations periodically transmit their routing tables to their immediate

Neighbors. a station also transmits its routing table if a significant change has occurred in its table from the last update sent. So, the update is both time-driven and event-driven. As stated above one of "full dump" or an incremental update is used to send routing table updates for reducing network traffic. A full dump sends the full routing table to the neighbors and could span many packets whereas in an incremental update only those entries from the routing table are sent that has a metric change since the last update and it must fit in a packet. If there is space in the incremental update packet then those entries may be included whose sequence number has changed. When the network is relatively stable, incremental updates are sent to avoid extra traffic and full dump are relatively infrequent. In a fast-changing network, incremental packets can grow big so full dumps will be more frequent [2].

Each route update packet, in addition to the routing table information, also contains a unique sequence number assigned by the transmitter. The route labeled with the highest (i.e. most recent) sequence number is used. If two routes have the same sequence number then the route with the best metric (i.e. shortest route) is used. Based on the past history, the stations estimate the settling time of routes. The stations delay the transmission of a routing update by settling time so as to eliminate those updates that would occur if a better route were found very soon. Each row of the update send is of the of the form <Destination IP address, Destination sequence number, Hop count $>$. After receiving an update neighboring nodes utilizes it to compute the routing table entries.

To damp the routing fluctuations due to unsynchronized nature of periodic updates, routing updates for a give destination can propagate along different paths at different rates. To prevent anode from announcing a routing path change for a given destination while another better update for that destination is still in route, DSDV 11 requires node to wait a settling time before announcing a new route with higher metric for a destination. 


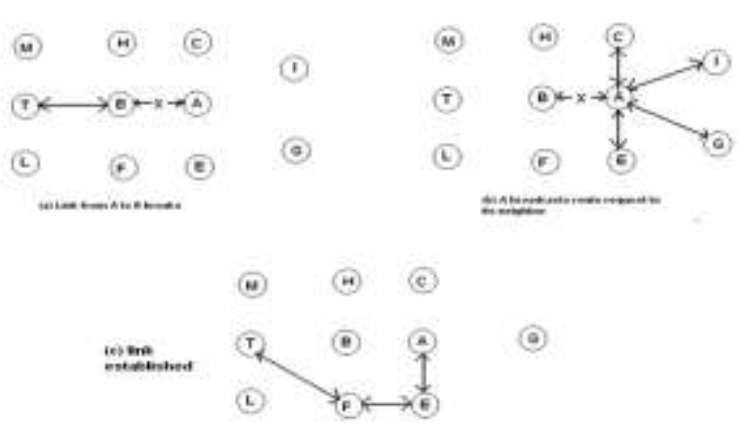

Figure 1

Resolving failed links in DSDV

IV. DSR

The Dynamic Source Routing protocol (DSR) is a simple and efficient routing protocol designed specifically for use in multi-hop wireless ad hoc networks of mobile nodes. DSR allows the network to be completely selforganizing and self-configuring, without the need for any existing network infrastructure or administration. Dynamic Source Routing, DSR, is a reactive routing protocol that uses source routing to send packets. It uses source routing which means that the source must know the complete hop sequence to the destination. Each node maintains a route cache, where all routes it knows are stored. The route discovery process is initiated only if the desired route cannot be found in the route cache. To limit the number of route requests propagated, a node processes the route request message only if it has not already received the message and its address is not present in the route record of the message.

As mentioned before, DSR uses source routing, i.e. the source determines the complete sequence of hops that each packet should traverse. This requires that the sequence of hops is included in each packet's header. A negative consequence of this is the routing overhead every packet has to carry. However, one big advantage is that intermediate nodes can learn routes from the source routes in the packets they receive. Since finding a route is generally a costly operation in terms of time, bandwidth and energy, this is a strong argument for using source routing. Another advantage of source routing is that it avoids the need for up-to-date routing information in the intermediate nodes through which the packets are forwarded since all necessary routing information is included in the packets. Finally, it avoids routing loops easily because the complete route is determined by a single node instead of making the decision hop-by-hop [3][4].

The protocol is composed of the two main mechanisms of "Route Discovery" and "Route Maintenance", which work together to allow nodes to discover and maintain routes to arbitrary destinations in the ad hoc network. All aspects of the protocol operate entirely on demand, allowing the routing packet overhead of DSR to scale automatically to only what is needed to react to changes in the routes currently in use. The protocol allows multiple routes to any destination and allows each sender to select and control the routes used in routing its packets, for example, for use in load balancing or for increased robustness.

\section{Route Discovery}

Route Discovery is used whenever a source node desires a route to a destination node. First, the source node looks up its route cache to determine if it already contains a route to the destination. If the source finds a valid route to the destination, it uses this route to send its data packets. If the node does not have a valid route to the destination, it initiates the route discovery process by broadcasting a route request message. The route request message contains the address of the source and the destination, and a unique identification number. An intermediate node that receives a route request message searches its route cache for a route to the destination. If no route is found, it appends its address to the route record of the message and forwards the message to its neighbors. The message propagates through the network until it reaches either the destination or an intermediate node with a route to the destination. Then a route reply message, containing the proper hop sequence for reaching the destination, is generated and uncast back to the source node. [4]

To verify the rules (policy) against the statements (reputation expressed in the credentials). The result of this process is either compliance or inconsistency. In the latter case, if the policy lists additional trusted entities, the compliance checker asks the credential manager to search for another chain of trust. Otherwise, a request denial or granted message is sent to the calling application. After the request is processed, the compliance checker updates a recommender-database. In this database, the TMS stores the recommender- reputations of all TTPs specified in its policies. After each transaction, the behavior of the entity involved in the transaction is rated. The discrepancy between this rating and the recommendation is determined and combined with previous 
experience to update the recommender reputation. To process the attributes that connect the policy via credentials to the requesting entity, the relevant interpreter module is called.

\section{Route maintenance}

Route Maintenance is used to handle route breaks. When a node encounters a fatal transmission problem at its data link layer, it removes the route from its route cache and generates a route error message. The route error message is sent to each node that has sent a packet routed over the broken link. When a node receives a route error message, it removes the hop in error from its route cache. Acknowledgment messages are used to verify the correct operation of the route links. In wireless networks acknowledgments are often provided as e.g. an existing standard part of the MAC protocol in use, such as the link-layer acknowledgment frame defined by IEEE 802.11. If a built in acknowledgment mechanism is not available, the node transmitting the message can explicitly request a DSR-specific software acknowledgment to be returned by the next node along the route. [4]

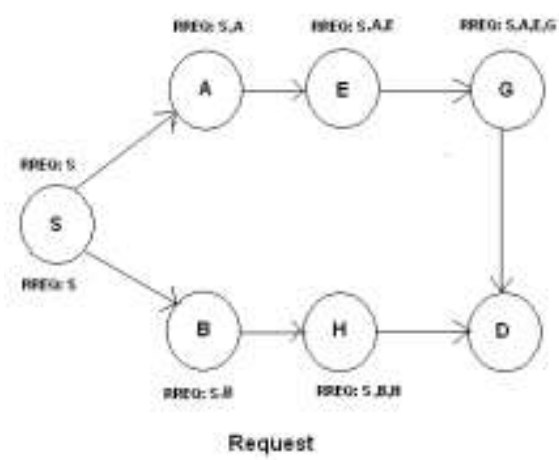

Figure 2

DSR request and Reply

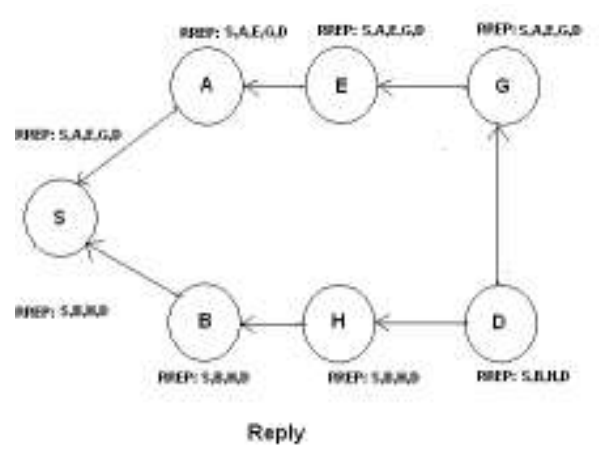

Figure 3

\section{PERFORMANCE METRICS}

The second goal of this project is to compare the performance of the two protocols under different scenario. Comparing the different methods is done by simulating them and examining their behavior. In comparing the two protocols, the evaluation could be done in the following three metrics:

1. The packet delivery ratio defined as the number of received data packets divided number of generated data packets.

2. The end to end delay is defined as the time a data packet is received by destination minus the time the data packet is generated by the source.

\section{PACKET DELIVERY RATIO}

Figure 4 shows packet delivery ratio with pause time varying from 2 to 10 for DSDV and DSR routing protocol. The red line shows graph for DSDV and the green line shows the graph for DSR protocol. The delivery ratio for both the protocols is always greater than 90 percent. The basic difference between the two protocols is very less. But generally the graph for the DSR protocol lies above than that of DSDV for most cases. However in certain cases the DSDV protocols is also better. It is more likely for the mobile nodes to have fresher and shorter routes to a gateway and thereby minimizing the risk for link breaks. Link breaks can result in lost data packets since the source continues to send data packets until it receives a RERR message from the mobile node that has a broken link. The longer the route is (in number of hops), the longer time it can take before the source receive a RERR and hence, more data packets can be lost. When the pause time interval increases, a mobile node receives less gateway information and consequently it does not update the route to the 
Gateway as often as for short advertisement intervals. Therefore, the positive effect of periodic gateway information is decreased as the advertisement interval increases.

\section{AVERAGE END TO END DELAY}

The average end-to-end delay is less for the DSDV approach than for the DSR approach. The reason is that the periodic gateway information sent by the gateways allows the mobile nodes to update their route entries for the gateways more often, resulting in fresher and shorter routes. With the DSR (reactive approach) a mobile node continues to use a route to a gateway until it is broken. In some cases this route can be pretty long (in number of hops) and even if the mobile node is much closer to another gateway it does not use this gateway, but continues to send the data packets along the long route to the gateway further away until the route is broken. Therefore, the end-to-end delay increases for these data packets, resulting in increased average end-to-end delay for all data packets. The average end-to-end delay is decreased slightly for short pause time intervals when the advertisement interval is increased. At the first thought this might seem unexpected. However, it can be explained by the fact that very short advertisement intervals result in a lot of control traffic which lead to higher processing times for data packets at each node.

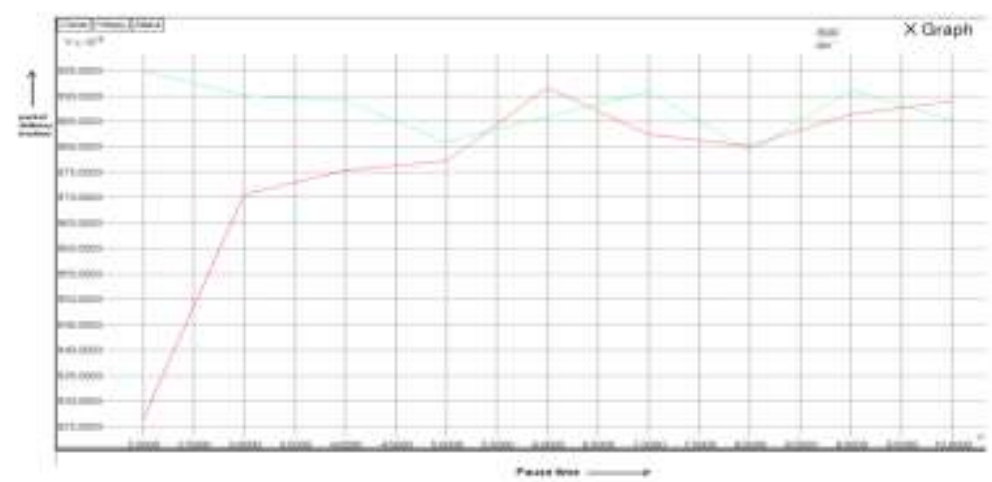

Figure 4

\section{CONCLUSION}

In this paper the Destination Sequenced Distance Vector and Dynamic Source Routing protocols in tool command language and integrated the module in the ns-2 Simulator. The performance of the protocols were measured with respect to metrics like Packet delivery ratio, end to end delay. I have made the performance comparison of the protocols and Simulations were carried out with identical topologies and running different protocols on the mobile node. The results of the simulation indicate that performance of the DSR protocol is superior to standard DSDV. It is also observed that the performance is better especially when the pause time is low. For higher pause time although DSR is better for most cases but their delivery ratio remains close to each other. It is also true that any of the single protocol does not supersede the other one. There performance depends upon the different scenarios.

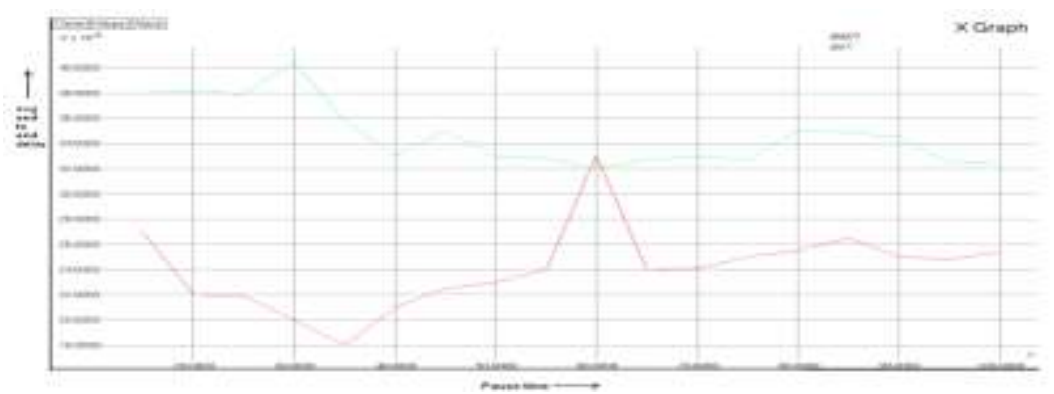

Figure 5

\section{REFERENCES}

[1]. D. Kim, J. Garcia and K. Obraczka, "Routing Mechanisms for Mobile Ad Hoc Networks based on the Energy Drain Rate", IEEE Transactions on Mobile Computing. Vol 2, no 2, 2003, pp.161-173

[2]. C.E. Perkins \& P. Bhagwat, "Highly Dynamic Destination Sequence-Vector Routing (DSDV) for Mobile Computers", Computer Communication Review, vol. 24, no.4, 1994, pp. 234-244.

[3]. C.E. Perkins and E.M. Royer, “Ad-Hoc on-Demand Distance Vector Routing,” Proc. Workshop Mobile Computing Systems and Applications (WMCSA '99), Feb. 1999 pp. 9

[4]. David B. Johnson And David Maltz. "Dynamic source routing in ad hoc wireless networks", Mobile Computing, Kluwer Academic Publishers. 1996 pp.153-181, 1996

[5]. M. S. Corson, J.P. Maker and G. H. Cirincione "Internet-Based Mobile Ad Hoc Networking," IEEE Interne Computing, Vol. 3, no. 4, July-August 1999, pp. 63-70. 